\title{
microRNA-183 is involved in the differentiation and regeneration of Notch signaling-prohibited hair cells from mouse cochlea
}

\author{
WEI ZHOU ${ }^{1,2 *}$, JINTAO DU $^{1,3^{*}}$, DI JIANG ${ }^{1,4}$, XIANREN WANG $^{1}$, KAITIAN CHEN $^{1}$, HAOCHENG TANG $^{1}$, \\ XUEMEI ZHANG ${ }^{1,5}$, HUI CAO ${ }^{1}$, LING ZONG $^{1,6}$, CHANG DONG $^{1}$ and HONGYAN JIANG ${ }^{1}$
}

${ }^{1}$ Department of Otolaryngology, The First Affiliated Hospital and Institute of Otorhinolaryngology, Sun Yat-sen University, Guangzhou, Guangdong 510080; ${ }^{2}$ Department of Otolaryngology, People's Hospital of Meishan, Meishan, Sichuan 620010;

${ }^{3}$ Department of Otolaryngology, West China Hospital of Sichuan University, Chengdu, Sichuan 6100041; ${ }^{4}$ Department of

Otolaryngology, People's Hospital of Dongguan, Dongguan, Guangdong 510080; ${ }^{5}$ Department of Otorhinolaryngology,

The Second Hospital of Hebei Medical University, Shijiazhuang, Hebei 050000; ${ }^{6}$ Department of Otorhinolaryngology,

The Second Affiliated Hospital, Guangzhou Medical University, Guangzhou, Guangdong 510260, P.R. China

Received March 19, 2017; Accepted December 5, 2017

DOI: $10.3892 / \mathrm{mmr} .2018 .9127$

\begin{abstract}
Auditory hair cell regeneration following injury is critical to hearing restoration. The Notch signaling pathway participates in the regulation of inner ear development and cell differentiation. Recent evidence suggests that microRNA (miR)-183 has a similar role in the inner ear. However, it is unclear how Notch signaling functions in hair cell regeneration in mammals and if there is cross-talk between Notch signaling and miR-183. The present study used a gentamicin-induced cochlear injury mouse model. Gentamicin-induced damage of the hair cells activated the Notch signaling pathway and downregulated miR-183 expression. Notch signaling inhibition by the $\gamma$-secretase inhibitor, 24-diamino-5-phenylthiazole (DAPT), attenuated gentamicin-induced hair cell loss and reversed the downregulation of miR-183 expression. Further investigation revealed that the novel hair cells produced, induced by DAPT, were derived from transdifferentiated supporting cells. Additionally, myosin VI-positive hair cell numbers were increased by Notch signaling inhibition in in vitro experiments with cultured neonatal mouse inner ear precursor cells. This effect was reversed by miR-183 inhibition. These findings indicate that the Notch signaling pathway served a repressing role during the regeneration of hair cells. Inhibiting this signal improved hair cell regeneration in the gentamicin-damaged
\end{abstract}

Correspondence to: Professor Hongyan Jiang, Department of Otolaryngology, The First Affiliated Hospital and Institute of Otorhinolaryngology, Sun Yat-sen University, 58 Zhongshan Road, Guangzhou, Guangdong 510080, P.R. China

E-mail: hongyanjiang@sina.com

*Contributed equally

Key words: hair cells, regeneration, Notch signaling, microRNA-183, gentamicin cochlear model. miR-183 was demonstrated to be involved in hair cell differentiation and regeneration, and was required for the differentiation of the Notch-inhibited hair cells.

\section{Introduction}

Non-mammalian vertebrates are able to regenerate auditory and vestibular hair cells following trauma to directly improve the functional recovery of hearing and balance $(1,2)$. However, in mammals, there is a limited capacity for spontaneous hair cell regeneration in the vestibular system and no spontaneous hair cell regeneration has been observed in the mature cochlea. Therefore, sensory hair cell damage is a major cause of mammalian hearing loss and balance disorder $(1,3)$.

The development of a functionally mature cochlea requires the engagement of a number of signaling pathways, including Notch, bone morphogenetic protein and Wnt. The Notch signaling pathway mediates hair and supporting cell differentiation during the later period of inner ear development, when Notch1, hes family basic helix-loop-helix transcription factor (Hes) 1 and Hes 5 are expressed in supporting cells (4-7). Therefore, it is clear that Notch signaling is crucial for inner ear differentiation and typical mosaic pattern formation. In mature mammals, hair cell regeneration may fail if supporting cell differentiation is inhibited. Hartman et al (8) and Murata et al (9) demonstrated that Notch signaling molecules were activated in a drug-damaged cochlea mouse model. Therefore, the Notch signaling pathway may be a climacteric pathway for the regeneration of hair cells and the dedifferentiation of supporting cells.

A previously identified microRNA (miR), miR-183, may have an important role in inner ear development and function (10). It has been previously demonstrated that during sensory epithelial differentiation, miR-183 is expressed in hair cells, whereas Notch1 and Hes1 are primarily expressed in supporting cells $(9,11)$. The spatially exclusive expression pattern of miR-183 and Notch1 during inner ear development suggests a potential association between miR-183 and Notch signaling. 
In the current study, gentamicin-treated cells had significantly reduced the number of myosin VI-positive hair cells in the post-neonatal mice explanted cochlear. Notch1 signaling in the supporting cells was also increased. Inhibition of Notch signaling by DAPT attenuated the gentamicin-induced hair cell loss. Conversely, the expression of the miR-183 cluster was downregulated following gentamicin treatment. This downregulation may be reversed by DAPT. It is of note, the increase in myosin VI-positive cells induced by DAPT was abolished by miR-183 inhibition.

\section{Materials and methods}

Animals. Post-natal day 1 (P1) C57BL/6 mice (n=480; average weight $1.0 \mathrm{~g}$ ) were obtained from the Experimental Animal Center of Sun Yat-sen University (Guangzhou, China). The study protocol was approved by the Institution Review Board of Sun Yat-sen University (Guangzhou, China). All animal experiments were performed within 2-3 h of the arrival of the mice and in compliance with the guidelines of the Animal Care and Use Committee of the National Institutes of Health of USA for experimental use of laboratory animals.

Organ and cell culture. Hank's balanced salt solution (HBSS, pH 7.4), supplements N2 (100x) and B27 (50x), Dulbecco's modified Eagle's medium/F12 (DMEM/F12) were purchased from Invitrogen (Thermo Fisher Scientific, Inc., Waltham, MA, USA). Collagen-coated cover slides, penicillin G, heparin sulfate, and bromodeoxyuridine (BrdU) were purchased from Sigma-Aldrich (Merck KGaA, Darmstadt, Germany). C57BL/6 mice were euthanized at postnatal day 1 and cochlear sensory epithelium was collected and dissected in HBSS. The stria vascularis, Reissner's membrane and the tectorial membrane were removed prior to transfer onto the collagen-coated cover slides. One group of organ samples from 20 mice were incubated in serum-free DMEM/F12 media supplemented with $\mathrm{N} 2, \mathrm{~B} 27$ and $100 \mathrm{U} / \mathrm{ml}$ penicillin G. Culture medium was changed every other day. Following 8 days culture the incubated cochleae were then fixed with $4 \%$ paraformaldehyde at room temperature for $30 \mathrm{~min}$.

The inner ear sensory epithelial sheets were isolated from the saccule and utricle of C57BL/6 mice. The otolith was carefully dissected under a stereoscopic microscope in a separate dish with ice-cold HBSS. The isolated inner ear sensory epithelial sheets were transferred into Eppendorf tubes, digested in $500 \mu \mathrm{l}$ of $0.125 \%$ trypsin in phosphate-buffered saline (PBS; Gibco; Themo Fisher Scientific, Inc.) at $37^{\circ} \mathrm{C}$ for $15 \mathrm{~min}$. The cells were carefully triturated with plastic $200 \mu \mathrm{l}$ pipette tips, centrifuged $(3,000 \mathrm{x} \mathrm{g}, 5 \mathrm{~min}$ at room temperature) and suspended in $2 \mathrm{ml}$ DMEM/F12 medium with N2 and B27 supplements, epidermal growth factor (EGF; $20 \mathrm{ng} / \mathrm{ml}$; Invitrogen; Thermo Fisher Scientific, Inc.), insulin-like growth factor 1 (IGF-1, $20 \mathrm{ng} / \mathrm{ml}$, PeproTech, Rocky Hill, NJ, USA), basic fibroblast growth factor (bFGF; $20 \mathrm{ng} / \mathrm{ml}$, R\&D Systems, Minneapolis, MN, USA). The dissociated cells were passed through a $70 \mu \mathrm{m}$ cell filter (BD Biosciences, Franklin Lakes, NJ, USA) to remove cell clumps. Half of the medium was exchanged every other day. The solid spheres were collected after 5 days of culture, transferred into chamber slides coated with Matrigel $^{\mathrm{TM}}$ (BD Biosciences), and allowed to cultivated up to 11 days in the same medium without growth factors. The inner ear sensory precursor cells were fixed with $4 \%$ paraformaldehyde at room temperature for $30 \mathrm{~min}$.

Drug treatment. In order to induce injury in hair cells, the isolated organs were incubated with $150 \mu \mathrm{M}$ gentamicin (Shanghai DingGuo Biotech Co., Ltd., Shanghai, China) for 14 h. DAPT (5 $\mu$ M, D5942; Sigma-Aldrich; Merck KGaA) or dimethyl sulfoxide (DMSO; $15 \mu \mathrm{M}$; Invitrogen; Thermo Fisher Scientific, Inc.; negative control) were subsequently added to substitute for gentamincin. The cochleae were fixed after 3.5 days for sex determining region Y-box 2 (Sox2; SAB2104660; 1:250; Sigma-Aldrich; Merck KGaA), anti-active Notch1 (1:200; ab8925), 4'6-diamidino-2-phenylindole (DAPI; 1:1,000; ab104139; both Abcam, Cambridge, UK) and myosin VI (1:200; 25-6791; rabbit polyclonal, Proteus Biosciences Inc., Ramona, CA, USA) staining. Organs were cultured for 8 days for myosin VI, active Notch1, anti-bromodeoxyuridine (anti-BrdU; 1:200; Sigma-Aldrich; Merck KGaA) Rhodamine-phalloidin (1:200; Invitrogen; Thermo Fisher Scientific, Inc.), and DAPI staining. For proliferation analysis, BrdU was added at a final concentration of $10 \mu \mathrm{M}$ following washes to remove gentamycin. The organs with BrdU and the control group were cultured for 3.5 days following the washing out of the gentamicin prior to staining. Inner ear precursor cells were incubated with $50 \mu \mathrm{M}$ DAPT as a negative control reagent, with or without small interfering RNA (siRNA) transfection of the miR-183 family (miR-183, miR-182, miR-96) by electroporation 5 days post-culture. The inner ear precursor cells continued to be cultured for 5 days.

Immunohistochemistry and microscopy. Immunostaining of the cultured organs of Corti were performed as previously described (12) using the following primary antibodies: Myosin VI, mouse monoclonal anti-BrdU, mouse monoclonal anti-active Notch1 and rabbit polyclonal anti-Sox2 (SAB2104660; 1:250; Sigma-Aldrich; Merck KGaA; all overnightat $4^{\circ} \mathrm{C}$ ). The following secondary antibodies were used: Alexa Fluor ${ }^{\circledR}$ 488-conjugated and Alexa Fluor ${ }^{\circledR}$ 594-conjugated donkey anti-rabbit, Alexa Fluor ${ }^{\circledR}$ 488-conjugated and Alexa Fluor ${ }^{\circledR}$ 594-conjugated donkey anti-mouse (1:400; R37118, R37119, R37114, R37115 respectively; Invitrogen; Thermo Fisher Scientific, Inc.). Following the fixation of the cultured organs, they were permeabilized in $0.1 \%$ Triton X-100 in PBS for $15 \mathrm{~min}$ and washed three times for $5 \mathrm{~min}$ in PBS. The organs were blocked in $5 \%$ bovine serum albumin (AR1006; Boster Biological Technology, Ltd., Wuhan, China)/0.05\% Triton $\mathrm{X}-100$ in PBS for $1 \mathrm{~h}$ prior to incubation with the primary antibodies overnight at $4^{\circ} \mathrm{C}$. Incubation with the secondary antibodies (1:400) was performed at $4^{\circ} \mathrm{C}$ overnight or at room temperature for $2 \mathrm{~h}$. Rhodamine-phalloidin was used to stain the stereocilium and reticular laminae of the cochlear hair cells for $30 \mathrm{~min}$ in room temperature. DAPI was used to stain the nucleus for $15 \mathrm{~min}$ at room temperature. Specimens were mounted and observed under a fluorescence microscope (Olympus IX71 Inverted Microscope; Olympus Corporation, Tokyo, Japan) and a FluoView FV1000 confocal microscope (Olympus Inc., Guangzhou, Guangdong, China). Specimens were examined at $\mathrm{x} 20$ or $\mathrm{x} 40$ magnification. Images were processed using Image-Pro Plus software version 6.0 
Table I. Primer sequences used for reverse transcription-quantitative polymerase chain reaction.

\begin{tabular}{lll}
\hline Gene & \multicolumn{1}{c}{ Sense (5'-3') } & \multicolumn{1}{c}{ Antisense (5'-3') } \\
\hline Atoh1 & AGATCT ACATCAACGCTCTGTC & ACTGGCCTCATCAGAGTCACTG- \\
Hes 1 & GGAGAGGCTGCCAAGGTTTT & GCAAATTGGCCGTCAGGA \\
Notch1 & GCTGACCTGCGCATGTCTGCCATG & CATGTTGTCCTGGATGTTGGCATCTG \\
Jagged 1 & CAGAATGACGCCTCCTGTCG & TGCAGCTGTCAATCACTTCG \\
Delta1 & TACTGCACTGACCCAATCTG & GGCACCGTTAGAACAAGG \\
p27 & CTGGAGCGGATGGACGCCAGAC & CGTCTGCTCCACAGTGCCAGC \\
GAPDH & AACGGGAAGCCCATCACC & CAGCCTTGGCAGCACCAG
\end{tabular}

Atoh1, atonal basic helix loop helix transcription factor 1; Delta1, delta like canonical Notch ligand 1; Hes1, hes family basic helix loop helix transcription factor 1; p27, cyclin-dependent kinase inhibitor $1 \mathrm{~B}$.

(Media Cybernetics, Inc., Rockville, MD, USA). Cultured precursor sensory epithelia were prepared for staining and fixed in 4\% PFA/PBS for $20 \mathrm{~min}$ at room temperature and then washed in PBS three times. Subsequently, permeabilization, blocking and antibody incubation were performed as described for organ samples.

For organ culture, the number of hair cells was determined by calculating the number of myosin VI-positive cells per $200 \mu \mathrm{m}$ in the organ of Corti. For cultured precursor sensory epithelia, the percentage of hair cells was determined by calculating the number of myosin VI-positive cells over the total number of cells in the whole sphere, multiplied by 100 . Cells positive for DAPI or myosin VI were counted in one representative optical section. Cells were only counted when their nucleus comprised $>50 \%$ of the cell area. Quantification was obtained from at least 6 random areas over the length of an entire cochlea. At least 5 or 6 inner ear precursor spheres were counted for each experiment.

Reverse transcription semi-quantitative polymerase chain reaction ( $R T$-sqPCR) analysis. The cultured organs (at day 8 ) were carefully dissected and collected in Eppendorf tubes. The total RNA was extracted and column-purified using RNeasy purification kit (Qiagen GmbH, Hilden, Germany) according to the manufacturer's protocol. Total RNA was treated with RNase-free DNase (Roche Diagnostics, Indianapolis, IN, USA). Reverse transcription was performed using SuperScript III one-step RT-PCR system with Platinum Taq High Fidelity DNA polymerase (1274-030; Invitrogen; Thermo Fisher Scientific, Inc.) according to the protocol provided by manufacturer. The PCR sequential steps are as follows: Denaturation at $94^{\circ} \mathrm{C}$ for $2 \mathrm{~min}$, annealing at $60^{\circ} \mathrm{C}$ for $30 \mathrm{sec}$, and extension at $72^{\circ} \mathrm{C}$ for $45 \mathrm{sec}$ : 35 cycles were performed and the last amplification was followed by a final 10-min elongation step at $72^{\circ} \mathrm{C}$. The primers (Invitrogen; Thermo Fisher Scientific, Inc.) used for PCR are presented in Table I. The PCR products were resolved by $1 \%$ agarose gel electrophoresis containing $0.4 \mu \mathrm{g} / \mathrm{ml}$ of ethidium bromide. The level of gene expression was semi-quantified by grey scale ratio of Atoh1, Notch1, Jagged1, Delta1, Hes1, P27 vs. GAPDH by Quantity One (version 4.6.2; Bio-Rad Laboratories, Inc. Hercules, CA, USA). Each experiment was performed (containing at least four different cochleae) at least three times from each group.
RT-quantitative (q)PCR (RT-qPCR) analysis of miR-183 family expression. RT-qPCR was performed based on a stem-loop Rtprimer design, as previously described (13). The cDNA was synthesized from extracted total RNA from cultured organs as mentioned above. Quantitative PCR (qPCR) was performed using $2 \mu \mathrm{l}$ of SYBR-Green/ROX qPCR Master Mix (Suzhou GenePharma Co., Ltd., Suzhou, China). All procedures were subjected to a TaqMan miRNA assay (Applied Biosystem 7500) according to the manufacturer's instructions. U6 was employed as an endogenous control to normalize data using the $2^{-\Delta \Delta \mathrm{Cq}}(14)$ method.

Probe and primers specific for miR-183, miR-182, miR-96, or for U6 RNA internal control were purchased from LC Sciences (Hangzhou, China). RT-qPCR analyses of at least 3 independent cultures were performed.

The qPCR reaction was performed using an ABI 7500 sequence detection system (Applied Biosystems; Thermo Fisher Scientific, Inc.) and used the following PCR protocol: $95^{\circ} \mathrm{C}$ for $10 \mathrm{~min}$ followed by 40 cycles of $95^{\circ} \mathrm{C}$ for $10 \mathrm{sec}$ and $55^{\circ} \mathrm{C}$ for $30 \mathrm{sec}$.

In situ hybridization. In situ hybridization procedure was performed as previously described (15). The cochleae explants were cultured in vitro for 8 days and subsequently fixed in fresh $4 \%$ paraformaldehyde at room temperature for $1-2 \mathrm{~h}$. Then the slides of cochleae were washed for $3 \times 5 \mathrm{~min}$ in 1 XPBS at room temperature. Followed proteinase $\mathrm{K}$ treatment, the slides were rinsed in 1XPBS at room temperature and placed horizontally in the hybridization chamber with $70 \mu$ l of hybridization buffer. Cochleae were prehybridized by incubating for 4-8 h at room temperature. Next, the cochleae were carefully pipetted with probed hybridization buffer and hybridized at $50-60^{\circ} \mathrm{C}$ overnight. The next day, the slides were soaked in prewarmed $60^{\circ} \mathrm{C} 5 \mathrm{XSSC}$ and then incubated in $0.2 \mathrm{XSSC}$ at $60^{\circ} \mathrm{C}$ for $1 \mathrm{~h}$. Finally, the slides were incubated in B1 solution $(0.1 \mathrm{M}$ Tris $\mathrm{pH} 7.5 / 0.15 \mathrm{M} \mathrm{NaCl}$ ) at room temperature for $10 \mathrm{~min}$ and then blocked in blocking solution ( $2 \mathrm{ml}$ of fetal calf serum, $18 \mathrm{ml}$ of $\mathrm{B} 1$ and $0.1 \%$ Tween-20) for $1 \mathrm{~h}$ at room temperature. The slides were carefully pipetted with blocking solution containing anti-DIG-alkaline phosphatase antibody (diluted 1:1,000), followed by incubation at $4^{\circ} \mathrm{C}$ overnight. The next day, the slides were washed three times for 5 min each time in B1 solution at room temperature. Then, cochleae were equilibrated for 


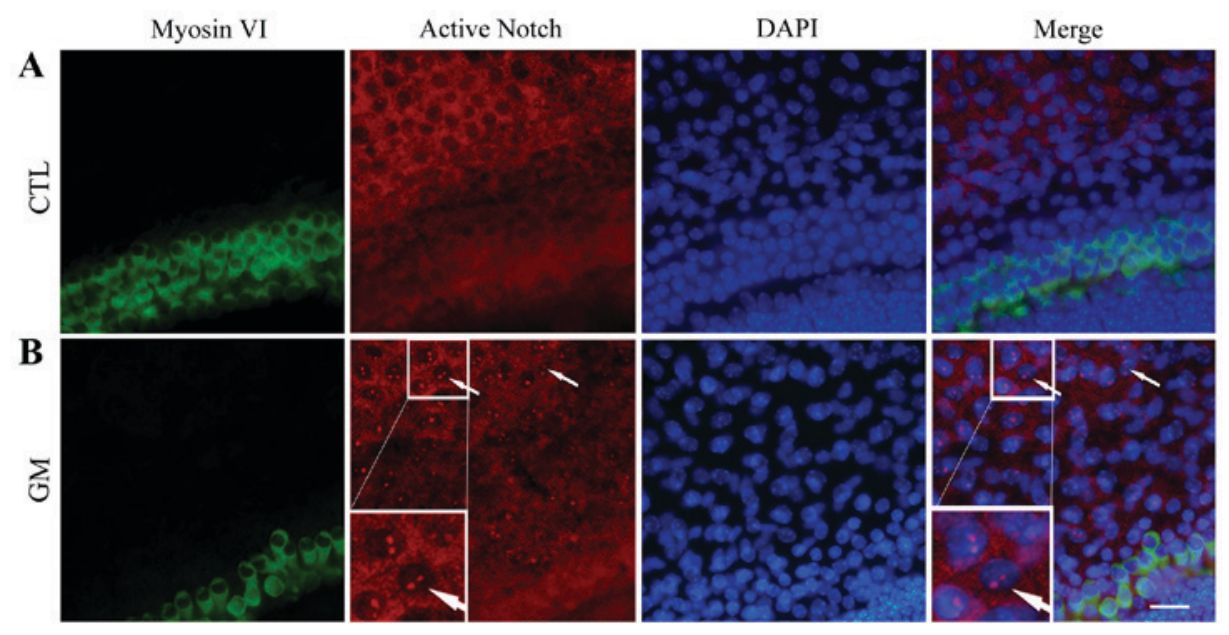

Figure 1. Gentamicin-induced injury of neonatal cochleae activates Notch signaling. Cochleae of postnatal day 1 mice were incubated (A) without gentamicin or (B) with $150 \mu \mathrm{M}$ gentamicin for $14 \mathrm{~h}$ and were immunostained with myosin VI (green) and activated Notch1 (red). The white arrow indicates positive activated Notch1. Nuclei were stained blue with DAPI. Scale bar, $20 \mu \mathrm{m}$. DAPI, 4'6-diamidino-2-phenylindole; GM, gentamicin; CTL, control.

10 min in B3 solution (0.1 M Tris pH 9.5/0.1 M NaCl/50 mM $\left.\mathrm{MgCl}_{2}\right)$. Finally, the developer solution $(3.4 \mu \mathrm{l}$ of $100 \mathrm{mg} / \mathrm{ml}$ NBT, $3.5 \mu \mathrm{l}$ of $50 \mathrm{mg} / \mathrm{ml}$ BCIP, $2.4 \mu \mathrm{l}$ of $24 \mathrm{mg} / \mathrm{ml}$ levamisole, $5 \mu \mathrm{l}$ of $10 \%$ Tween and $986 \mu \mathrm{l}$ of B3) was carefully added to the slides, which were kept in a humidified chamber. The reaction was developed at room temperature in the dark for times ranging from 1-3 days, depending on the microRNA expression levels. A light microscope (Leica Microsystems $\mathrm{GmbH}$, Wetzlar, Germany) was used to monitor the reaction, which was terminated when a strong blue stain was observed. Washing the slides for $3 \times 10$ min in 1 XPBT terminated the color reaction. The slides were mounted in fluorescent mounting media (S3023; Dako; Agilent Technologies, Inc., Santa Clara, CA, USA) for further observation.

Electroporation. The siRNA oligoribonucleotides for the miR183 family (miR-183, miR-182, miR-96) were purchased from Shanghai GenePharma Co., Ltd., (Shanghai, China), cultured inner ear precursor cells were dissociated into a single-cell suspension with $0.25 \%$ trypsin (Gibco; Thermo Fisher Scientific, Inc.) at $37^{\circ} \mathrm{C}$ for $3 \mathrm{~min}$. Then, $1 \times 10^{6}$ cells/well were transfected by electroporation with $5 \mu \mathrm{g}$ miR-183 siRNA oligoribonucleotides, according to the manufacturer's protocol. An enhanced green fluorescent protein RNA oligoribonucleotide (Shanghai GenePharma Co., Ltd.) was used to monitor the efficiency of the electroporation. The transfected inner ear precursor cells were plated on cover glasses coated with poly-L-lysine and laminin (R\&D Systems, Inc.) and fixed with $4 \%$ paraformaldehyde at room temperature for $30 \mathrm{~min}$ following an additional 5 days culture. The inner ear precursor spheres with the average diameter of $55 \mu \mathrm{m}$ were selected for statistical evaluation.

Statistical analysis. Data are expressed as the mean \pm standard deviation and analyzed using SPSS version 13.0 (SPSS Inc., Chicago, IL, USA). One-way analysis of variance and Tukey's Honest Significant Difference was used as a post-hoc test in order to determine significant differences in multiple comparisons. $\mathrm{P}<0.05$ was considered to indicate a statistically significant difference.

\section{Results}

Gentamicin-induced injury of neonatal cochlear hair cells activates Notch signaling. To investigate the expression of activated Notch in hair and supporting cells, Notch1 expression was determined in control and gentamycin-damaged neonatal cochlear explants. An activated signal was not detected in control cochlear hair cells and supporting cells (Fig. 1A). However, fluorescence was detected in the nucleus of the supporting cells in the gentamicin-damaged cochleae, indicating an activated Notch signal (Fig. 1B).

Inhibition of Notch expression promotes postnatal cochlea hair cell differentiation and attenuates gentamicin-induced hair cell loss. Notch inhibition by DAPT $(5-20 \mu \mathrm{M})$ increased the number of myosin VI-positive cells in cochlear explants (Figs. 2 and 3) in a dose-dependent manner, with the most evident increase observed in the $20 \mu \mathrm{M}$ DAPT group $(\mathrm{P}<0.05$; Fig. 2E). The majority of the increase in hair cell number occurred at the apex turn, with limited changes in the basal turn (Fig. 2E). The ability of DAPT stimulate new hair cell growth in damaged postnatal cochlea was also examined. The results revealed that gentamicin treatment reduced the number of myosin VI-positive hair cells (Fig. 3C, G and J) compared with the controls (Fig. 3A, E and I). This reduction was mostly confined to the basal and middle turn of the cochleae, with virtually no hair cell loss occurring in the apex turn. Co-treatment of gentamicin and DAPT resulted in a significant increase in the number of myosin VI positive cells $(\mathrm{P}<0.05$; Fig. 3D, H and L) compared with gentamicin treatment alone. No hair cell loss was observed in the gentamicin-treated apex turn of the cochleae. DAPT and gentamicin treatment markedly increased the number of hair cells compared with gentamicin treatment alone (Fig. 3M; P<0.05). Additionally, DAPT treatment and gentamincin and DAPT co-treatment resulted in the blurring of the Corti's tunnel between inner and outer hair cells and crowding in the apex and middle turns due to new hair cell growth (Fig. 3B, D, F and H). Hair cells in the sensory epithelium region displayed stereocilia with varying degrees of disarrangement and random orientation (Fig. 3D, H and L). 


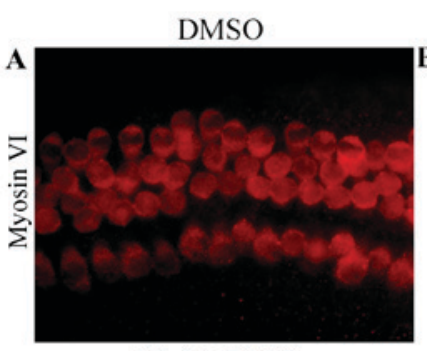

$10 \mu \mathrm{M}$ DAPT

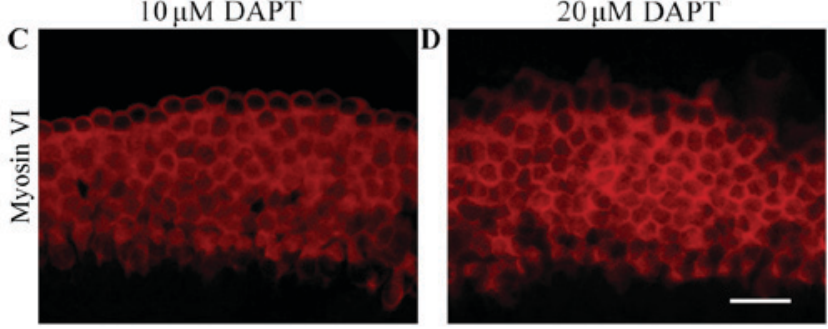

E

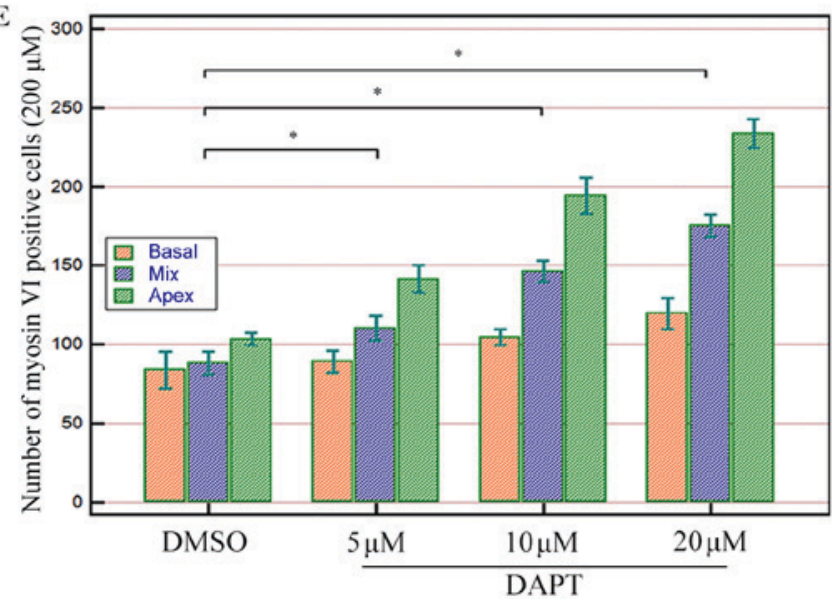

Figure 2. Notch inhibition induced hair cell differentiation in cochlear explants. Hair cells were incubated with (A) DMSO, (B) 5, (C) 10 or (D) $20 \mu \mathrm{M}$ DAPT. New hair cells were immunostained with anti-myosin VI antibodies (red). (E) Myosin VI-positive cells were counted. Data are presented as the mean \pm standard deviation, derived from at least 3 independent experiments. ${ }^{*} \mathrm{P}<0.05$. Scale bar, $20 \mu \mathrm{m}$. DAPT, 24-diamino-5-phenylthiazole; DMSO, dimethyl sulfoxide.

New hair cells are derived from transdifferentiated supporting cells. Activated Notch was revealed to be confined to the supporting cells of gentamicin-damaged cochleae (Fig. 1B). Thus, it was investigated whether hair cell proliferation was induced in response to DAPT treatment. The majority of BrdU-positive nuclei were observed in the area near outer hair cell and thus may be Hensen cells, according to the inner ear anatomy of mice. No BrdU and myosin VI double-positive cells were identified under any treatment conditions (Fig. 4A-D). Sox2, a marker of supporting cells, and myosin VI co-expressing cells were observed within the sensory epithelium in the DAPT and gentamicin co-treatment group (Fig. 4E). This suggested that the newly generated hair cells were derived from the differentiated, but not proliferating, supporting cells.

Notch signaling in the postnatal cochlea following gentamicin and DAPT co-treatment. RT-sqPCR results revealed that gentamicin-induced injury was associated with the upregulation of Notch1, Jagged1 and Hes1 expression and downregulation of Atoh1 expression (Fig. 5A). The expression of Jagged1 and Hes1 was downregulated in cochlear explants treated with DAPT. No apparent changes were observed in Delta1 or p27 expression, regardless of gentamicin or DAPT treatment. These results indicate that DAPT efficiently downregulated Notch signaling.

DAPT attenuates the downregulation of miR-183 family expression in gentamicin-damaged cochlear explants. Previous studies revealed that the miR-183 family (miR-183, miR-182, miR-96) is expressed in differentiated hair cells in spatially exclusive pattern with Notch1 (9-11). To investigate the association between the miR183 cluster and hair cell differentiation and regeneration in damaged cochlea, the expression of the miR183 cluster was examined in cochlea following gentamicin-induced injury. The same pattern of miR-183 cluster expression was observed in all groups (Fig. 5B). miR-183 cluster expression was significantly downregulated $(\mathrm{P}<0.05)$ in gentamicin-treated cochleae, whereas DAPT treatment significantly increased miR-183 cluster expression alone and in the presence of gentamicin (Fig. 5B; $\mathrm{P}<0.05$ ). Furthermore, in situ hybridization with LNA probes revealed similar results to the RT-qPCR analysis, demonstrating that DAPT treatment markedly increased miR-183 cluster expression, with or without gentamicin co-treatment (Fig. 5C-F). These findings suggest that the miR-183 cluster may play a role in DAPT-induced hair cell differentiation and regeneration.

MiR-183 is required for hair cell differentiation when the Notch signal is inhibited. Electroporation transfection efficiency was detected by eGFP protein with fluorescence microscopy $24 \mathrm{~h}$ after eGFP transfection (Fig. 6A). The cultured organs of Corti were repeatedly electroporated with miR-183 cluster inhibitors and eGFP fluorescence was not observed $24 \mathrm{~h}$ post-transfection. Therefore, inner ear precursor cells from neonatal mice were cultured. Cultured inner ear precursor cells were subsequently transfected with miR-183 cluster siRNA oligoribonucleotides. Transfection efficiency was determined to be $40-50 \%$ by eGFP. After 5 days of DAPT treatment, the number of myosin VI-positive cells was significantly increased (Fig. 6C) compared with controls $(\mathrm{P}<0.05$; Fig. 6B). However, the number of myosin VI-positive cells observed in the inner ear precursor cells pretreated with DAPT and miR-183 siRNA (Fig. 6D) was below that of the controls (Fig. 6B; $\mathrm{P}<0.05$ ), indicating that the DAPT-induced increase in myosin VI-positive cell numbers was abolished by miR-183 inhibition (Fig. 6, $\mathrm{P}<0.05)$. These results suggest that miR-183 is required for hair cell differentiation in Notch signaling-prohibited inner ear precursor cells.

\section{Discussion}

In mature mammals, hair cell regeneration may fail if supporting cells are inhibited from dedifferentiating. The Notch signaling pathway participates in the dedifferentiation of supporting cells via multiple mechanisms (16-19). In current study, the expression levels of activated Notch1, Jagged1 and Hes1 were increased, whereas Atoh1 was decreased in 

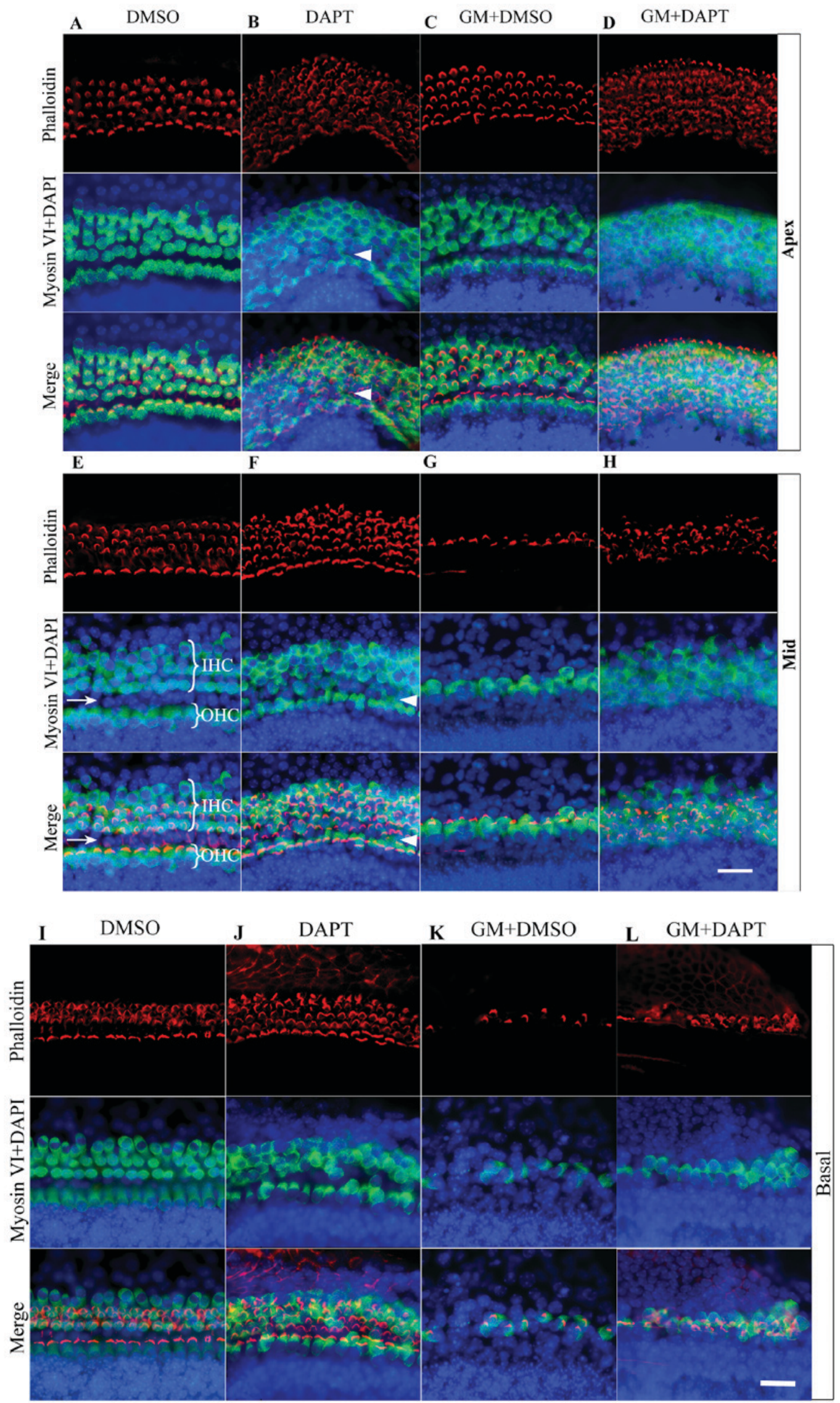

Figure 3. Notch inhibition promoted hair cell growth in normal and impaired cochleae. The apex turn of cochleae of post-natal day 1 mice were treated with (A) DMSO, (B) $10 \mu \mathrm{M}$ DAPT, (C) $150 \mu \mathrm{M}$ gentamicin and DMSO or (D) gentamicin and DAPT. The middle turn of cochlear of post-natal day 1 mice were treated with (E) DMSO, (F) $10 \mu \mathrm{M}$ DAPT, (G) $150 \mu \mathrm{M}$ gentamicin and DMSO or (H) gentamicin and DAPT. The basal turn of cochleae of post-natal day 1 mice were treated with (I) DMSO, (J) $10 \mu \mathrm{M}$ DAPT, (K) $150 \mu \mathrm{M}$ gentamicin and DMSO or (L) gentamicin and DAPT. Cochleae were immunostained with phalloidin antibodies (red) and anti-myosin VI antibodies (green). Nuclei were stained blue with DAPI. White brackets indicate the area of OHCs and IHCs. The white arrows indicate the tunnel of Corti. New hair cells appeared in the area of the sensory epithelia and occupied the tunnel of Corti region, as indicated by the white arrowheads. 
$\mathbf{M}$

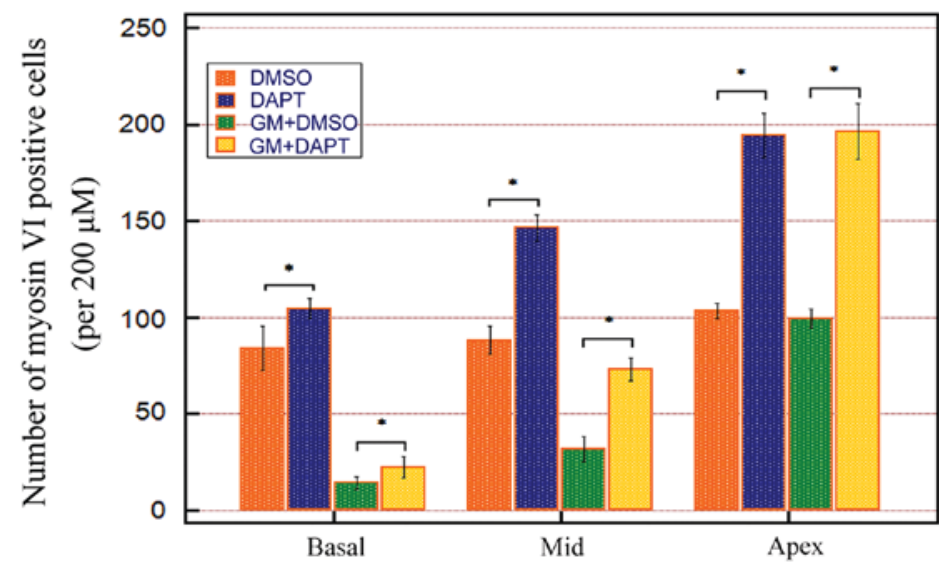

Figure 3. Continued. (M) Number of myosin VI-positive cells were counted. Data are presented as the mean \pm standard deviation, derived from at least 3 independent experiments. "P<0.05. Scale bar, $20 \mu \mathrm{m}$. GM, gentamicin; DMSO, dimethyl sulfoxide; DAPT, 24-diamino-5-phenylthiazole; IHC, inner hair cell; OHC, outer hair cell.

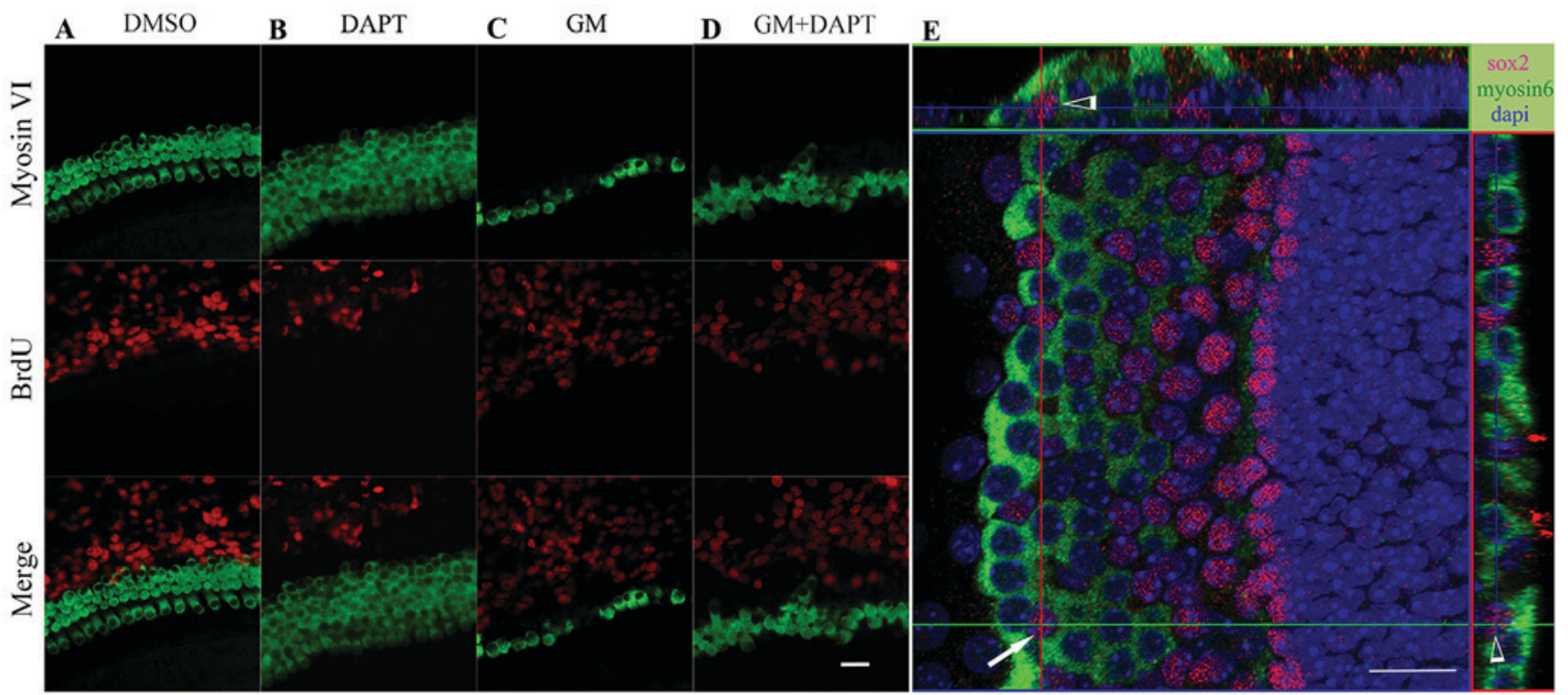

Figure 4. New hair cells following Notch inhibition are derived from supporting cell transdifferentiation, but not proliferation. Cochleae of post-natal day 1 mice were treated with (A) DMSO, (B) $10 \mu \mathrm{M}$ DAPT, (C) $150 \mu \mathrm{M}$ gentamicin or (D) gentamicin and DAPT and were immunostained with myosin VI (green), BrdU (red) and anti-Sox2 antibodies (purple). Nuclei were stained blue with DAPI. BrdU positive nuclei were identified in supporting cells, particularly in Hensen cells. No BrdU and myosin VI co-expressing cells were identified. (E) Sox2 and myosin VI were co-expressed (white arrow) in DAPT and gentamicin co-treatment group, as indicated by the white arrow. The hollow arrowheads indicate Sox 2 and myosin VI co-expressing cells on the $\mathrm{Y}$ and $\mathrm{Z}$ axis. Scale bar, $20 \mu \mathrm{m}$. GM, gentamicin; DMSO, dimethyl sulfoxide; DAPT, 24-diamino-5-phenylthiazole; BrdU, bromodeoxyuridine; Sox 2, sex determining region Y-box 2.

gentamicin-damaged cochleae. Activated Notch1 expression was also identified to be confined to the supporting cells of gentamicin-damaged cochleae. The new hair cells which were identified when Notch1 was inhibited by DAPT were derived from the supporting cells, rather than the mitosis or proliferation of existing hair cells. These findings suggest that Notch signaling plays an important role as a repressor of inner ear hair cell regeneration following gentamicin-induced damage. It is believed that hair cell regeneration in avian species occurs in two ways, by quiescent supporting cells directly differentiating into new hair cells or cells may divide first and subsequently differentiate into new hair and supporting cells $(1,6,20)$. The BrdU incorporation assay in the present study revealed that no BrdU expression was present in regenerated hair cells. Furthermore, Sox 2, a protein marker of supporting cells and myosin VI, a marker of hair cells, were co-expressed in certain hair cell-like cells. These findings support the hypothesis that regenerated hair cells do not come from mitosis but originate from supporting cell transdifferentiation in impaired mice cochlea in vitro. Hair cells and supporting cells share common precursors during inner ear development and avian hair cell regeneration comes from the supporting cells $(1,16)$. However, the specific regulatory mechanisms underlying these processes are not clear. Hair cell production in the tunnel of Corti following DAPT treatment in the present study suggested that pillar cells may be latent precursor cells. Pillar cells are a type of supporting cell which are found adjacent to hair cells and may be able to easily substitute the damaged hair cell following successful transdifferentiation. Misaligned regenerated hair cells, disordered stereocilia and no exact mosaic structure 

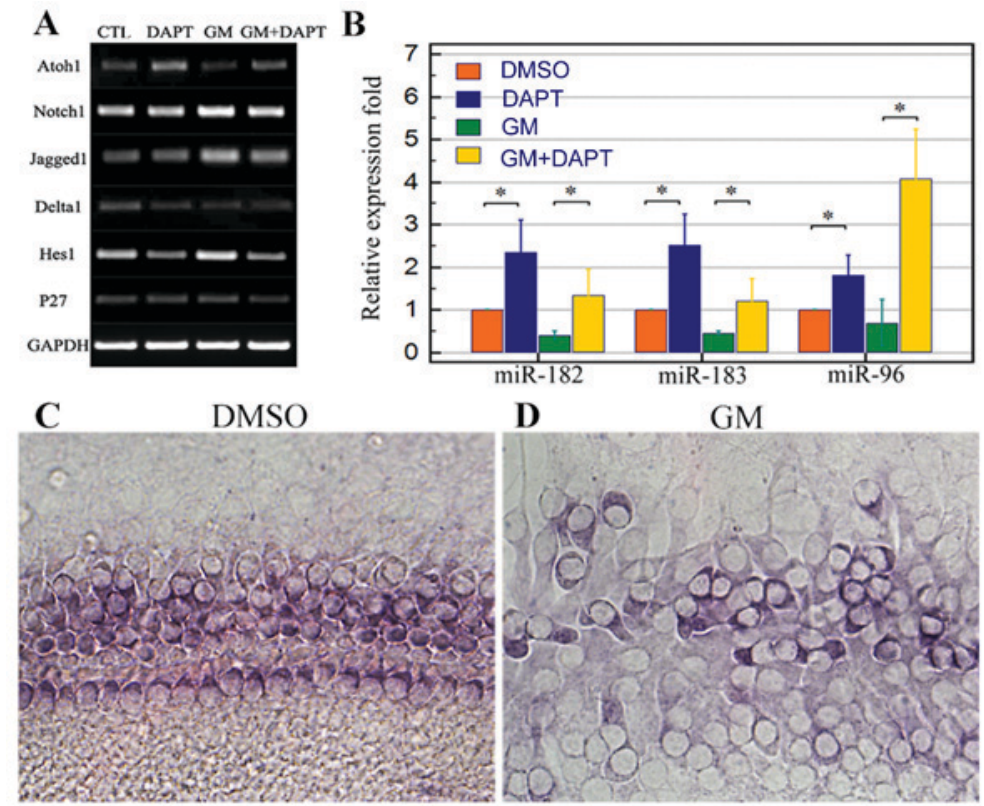

D

GM

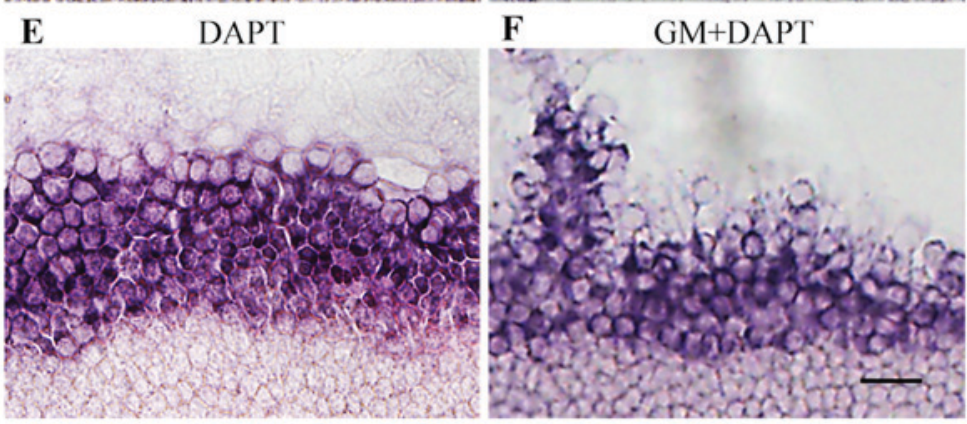

Figure 5. DAPT treatment suppressed Notch signaling in cochlear explants and stimulated miR-183 cluster expression. (A) Cochleae of post-natal day 1 mice were treated with DMSO, $10 \mu \mathrm{M}$ DAPT, $150 \mu \mathrm{M}$ gentamicin or gentamicin and DAPT. Downstream Notch signaling targets, including Atoh1, Notch1, Jagged1, Delta1, Hes1 and p27 were examined by RT-sqPCR. (B) miR-183 cluster expression was examined by RT-qPCR. Data are expressed as the mean \pm standard deviation, derived from at least 3 independent experiments. ${ }^{*} \mathrm{P}<0.05$. miR-183 expression was detected by in situ hybridization in the cochleae of post-natal day 1 mice treated with (C) DMSO, (D) $10 \mu \mathrm{M}$ DAPT, (E) $150 \mu \mathrm{M}$ gentamicin or (F) gentamicin and DAPT. DMSO, dimethyl sulfoxide; DAPT, 24-diamino-5-phenylthiazole; RT-qPCR, reverse transcription-quantitative polymerase chain reaction; RT-sqPCR, reverse transcription-semiquantitative polymerase chain reaction; miR, microRNA; Atoh1, atonal basic helix loop helix transcription factor 1; Delta1, delta like canonical Notch ligand 1; Hes1, hes family basic helix loop helix transcription factor 1; p27, cyclin-dependent kinase inhibitor 1B; GM, gentamicin.

were identified in explanted cochlear co-treated with gentamicin and DAPT. Additionally, the number of regenerated hair cells seemed to be proportional to the number of residual hair cells. Taken together, the results of the current study indicate that multiple signaling pathways may be involved in hair cell regeneration following cochlea injury. Future studies should be focused on the interaction between multiple signaling pathways that render structure formation and innervation between hair cells, the cochlear nerve and functional hair cell regeneration.

The miR-183 family regulates the sensorineural fates in the inner ear and is important for hair cell maintenance and survival (10). miR-183 expression is upregulated during inner ear progenitor cell differentiation into a hair cell-like fate. Ectopic miR-182 promotes inner ear progenitor cell differentiation into a hair cell-like fate (21). In the current study, Notch signaling suppression with DAPT promoted the numbers of myosin VI positive hair cells and upregulated miR-183 in cultured cochlea. Similarly, an increase in hair cell numbers was also observed with DAPT in inner ear precursor cells. Meanwhile, miR-183 siRNA was demonstrated to abolish the increase in hair cells induced by DAPT. No evident changes were detected in hair cells treated with miR-96 and miR-182 siRNA (data not shown). As Notch suppression may induce the formation of novel hair cells through the transdifferentiation of supporting cells, the aforementioned results suggested that miR-183 may be involved in the transdifferentiation of Notch signaling-inhibited hair cells. Furthermore, the activity of miR-183 in DAPT-induced hair cell regeneration was examined. The results revealed that Notch1 signaling was activated in gentamicin-injured hair cells and was accompanied by a reduction of miR-183 cluster expression. Suppression of Notch signaling by DAPT not only inhibited Notch signaling activation, but also upregulated miR-183 cluster expression which encouraged hair cell regeneration, indicating that the miR-183 cluster may be involved in hair cell regeneration induced by Notch suppression in gentamicin-treated cochleae. miR-183 cluster expression decreased following gentamicin-induced injury of the cochlea and was accompanied by Notch signaling activation. DAPT inhibition of Notch attenuated these effects. Additionally, miR-183 inhibition blocked the increase in 


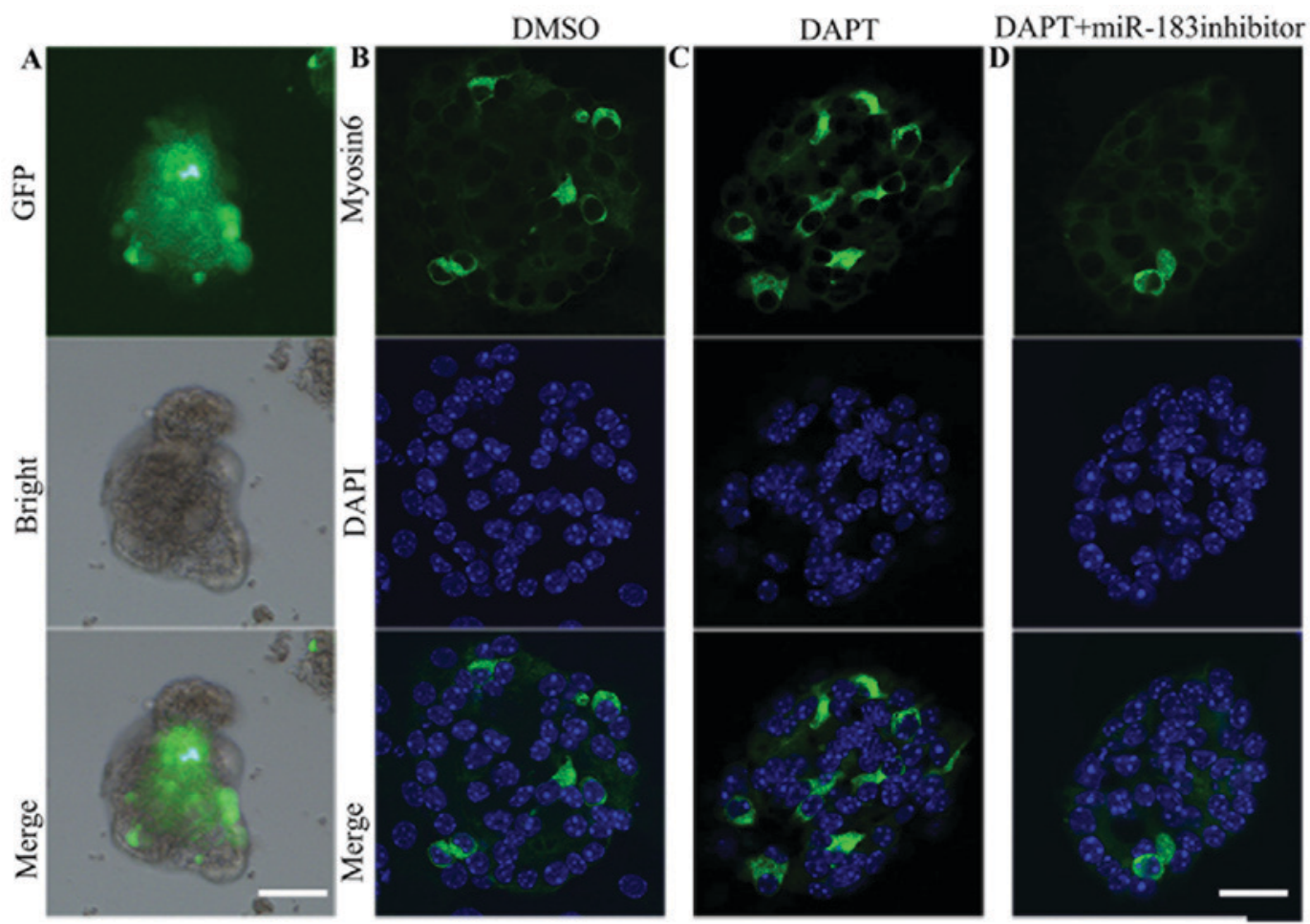

E

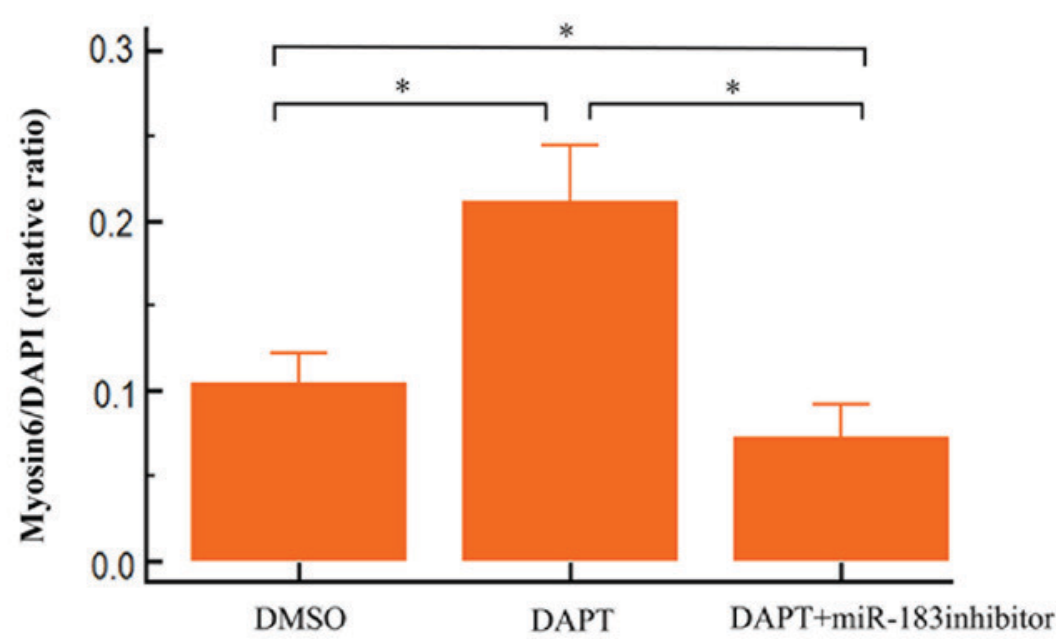

Figure 6. miR-183 was required for hair cell differentiation in the Notch-prohibited inner ear precursor cells. (A) GFP expression in the inner ear precursor cells following transfection with miR-183 small interfering RNA under a Leica light microscope. Inner ear precursor cells were treated with (B) DMSO, (C) $10 \mu \mathrm{M}$ DAPT or (D) DAPT and $100 \mathrm{nM}$ miR-183 inhibitor and were immunostained with anti-myosin VI antibodies (green). Nuclei were stained blue with DAPI. (E) Ratio of myosin VI-positive cells to DAPI-positive cells was calculated. Data are expressed as the mean \pm standard deviation, derived from at least 3 independent experiments. "P<0.05. Scale bar, $20 \mu \mathrm{m}$. miR, microRNA; DMSO, dimethyl sulfoxide; DAPT, 24-diamino-5-phenylthiazole; GFP, enhanced green fluorescent protein; GM, gentamicin.

hair cells induced by DAPT. The Notch signal pathway was not directly identified as a target of miR-183 in the present study, but a previous study revealed that the miR-183 family is associated with the Notch signaling pathway (22). Thus, further experiments to determine the detailed mechanism of miR-183 in hair cell differentiation are required. The present in vitro study used cultured neonatal mouse cochlea and inner ear precursor to investigate hair cell regeneration and differentiation. In vitro study is simple and convenient but many factors, including the influence of the environment and temperature are quite different from an in vivo experiment. As the neonatal cochlea, particularly the apex, still retains the ability to differentiate into hair cells (16), the data from the present study may not be strong enough to confirm that the hair cells in apex turn were regenerated from the gentamicin damaged inner ear cells. Therefore, future in vivo experiments are required.

In summary, the Notch signaling pathway was demonstrated to have an important role in the negative regulation of hair cell regeneration. Additionally, gentamicin-induced Notch activation reduced miR-183 expression. Inhibition of the Notch signaling pathway by DAPT attenuated these effects and promoted the transdifferentiation of supporting cells into hair cells. miR-183 may be a crucial factor in the differentiation and regeneration of the Notch signaling-inhibited hair cells from mouse cochlea. Inhibition of the Notch signaling 
pathway induced miR-183 expression, which participated in hair cell differentiation and regeneration. These findings suggest a therapeutic approach to promote mammalian hair cell regeneration by modulating Notch or miR-183 function.

\section{Acknowledgements}

The present study was supported by the grants from the National Basic Research Program of China (grant no. 2011CB504502), National Natural Science Foundation of China (grant no. 30973306, 81271076, 81200748 and 81500802), China Post-Doctoral Science Foundation (grant no. 2014M562329) and Key Natural Science Foundation of Guangdong Province (grant no. 8251008901000016).

\section{Competing interests}

The authors declare that they have no competing interests.

\section{References}

1. Corwin JT and Cotanche DA: Regeneration of sensory hair cells after acoustic trauma. Science 240: 1772-1774, 1988.

2. Ryals BM and Rubel EW: Hair cell regeneration after acoustic trauma in adult Coturnix quail. Science 240: 1774-1776, 1988.

3. Warchol ME, Lambert PR, Goldstein BJ, Forge A and Corwin JT: Regenerative proliferation in inner ear sensory epithelia from adult guinea pigs and humans. Science 259: 1619-1622, 1993.

4. Adam J, Myat A, Le Roux I, Eddison M, Henrique D, Ish-Horowicz D and Lewis J: Cell fate choices and the expression of Notch, Delta and Serrate homologues in the chick inner ear: Parallels with drosophila sense-organ development. Development 125: 4645-4654, 1998.

5. Daudet N and Lewis J: Two contrasting roles for Notch activity in chick inner ear development: Specification of prosensory patches and lateral inhibition of hair-cell differentiation. Development 132: 541-551, 2005.

6. Daudet N, Gibson R, Shang J, Bernard A, Lewis J and Stone J: Notch regulation of progenitor cell behavior in quiescent and regenerating auditory epithelium of mature birds. Dev Biol 326: 86-100, 2009.

7. Kelley MW: Regulation of cell fate in the sensory epithelia of the inner ear. Nat Rev Neurosci 7: 837-849, 2006.

8. Hartman BH, Basak O, Nelson BR, Taylor V, BerminghamMcDonogh O and Reh TA: Hes5 expression in the postnatal and adult mouse inner ear and the drug-damaged cochlea. J Assoc Res Otolaryngol 10: 321-340, 2009.
9. Murata J, Ikeda $\mathrm{K}$ and Okano $\mathrm{H}$ : Notch signaling and the developing inner ear. Adv Exp Med Biol 727: 161-173, 2012.

10. Weston MD, Pierce ML, Jensen-Smith HC, Fritzsch B Rocha-Sanchez S, Beisel KW and Soukup GA: MicroRNA-183 family expression in hair cell development and requirement of microRNAs for hair cell maintenance and survival. Dev Dyn 240: 808-819, 2011.

11. Wang XR, Zhang XM, Du J and Jiang H: MicroRNA-182 regulates otocyst-derived cell differentiation and targets T-box1 gene. Hea Res 286: 55-63, 2012.

12. Sage C, Huang M, Karimi K, Gutierrez G, Vollrath MA, Zhang DS, García-Añoveros J, Hinds PW, Corwin JT, Corey DP and Chen ZY: Proliferation of functional hair cells in vivo in the absence of the retinoblastoma protein. Science 307: 1114-1118, 2005.

13. Chen C, Ridzon DA, Broomer AJ, Zhou Z, Lee DH, Nguyen JT, Barbisin M, Xu NL, Mahuvakar VR, Andersen MR, et al: Real-time quantification of microRNAs by stem-loop RT-PCR. Nucleic Acids Res 33: e179, 2005.

14. Livak KJ and Schmittgen TD: Analysis of relative gene expression data using real-time quantitative PCR and the 2(-Delta Delta $\mathrm{C}(\mathrm{T})$ ) method. Methods 25: 402-408, 2001.

15. Kloosterman WP, Wienholds E, de Bruijn E, Kauppinen S and Plasterk RH: In situ detection of miRNAs in animal embryos using LNA-modified oligonucleotide probes. Nat Methods 3: 27-29, 2006.

16. Stone JS and Cotanche DA: Hair cell regeneration in the avian auditory epithelium. Int J Dev Biol 51: 633-647, 2007.

17. Stone JS and Rubel EW: Delta1 expression during avian hair cell regeneration. Development 126: 961-973, 1999.

18. Eddison M, Le Roux I and Lewis J: Notch signaling in the development of the inner ear: Lessons from Drosophila. Proc Natl Acad Sci USA 97: 11692-11699, 2000.

19. Żak M, Klis SF and Grolman W: The Wnt and Notch signalling pathways in the developing cochlea: Formation of hair cells and induction of regenerative potential. Int J Dev Neurosci 47: 247-258, 2015.

20. Roberson DW, Alosi JA and Cotanche DA: Direct transdifferentiation gives rise to the earliest new hair cells in regenerating avian auditory epithelium. J Neurosci Res 78: 461-471, 2004.

21. Wang XR, Zhang XM, Du J and Jiang H: MicroRNA-182 regulates otocyst-derived cell differentiation and targets T-box1 gene. Hear Res 286: 55-63, 2012.

22. Cheung CC, Lun SW, Chung GT, Chow C, Lo C, Choy KW and Lo KW: MicroRNA-183 suppresses cancer stem-like cell properties in EBV-associated nasopharyngeal carcinoma. BMC Cancer 16: 495, 2016.

This work is licensed under a Creative Commons Attribution-NonCommercial-NoDerivatives 4.0 International (CC BY-NC-ND 4.0) License. 